\title{
Ion-channel Sensors Based on ETH 1001 Ionophore Embedded in Charged- alkanethiol Self-assembled Monolayers on Gold Electrode Surfaces
}

\author{
Hiroshi Aoki, ${ }^{* \dagger}$ Yoshio Umezawa,* Alberto Vertova, ${ }^{* *}$ and Sandra RondinINI** \\ *Department of Chemistry, School of Science, The University of Tokyo, \\ 7-3-1 Hongo, Bunkyo, Tokyo 113-0033, Japan \\ **Department of Physical Chemistry and Electrochemistry, University of Milan, via Golgi 19, 20133 Milan, Italy
}

\begin{abstract}
An ion-channel sensor was demonstrated by immobilizing ETH 1001, an ionophore for ion-selective electrodes, on a gold electrode surface. The approach for preparing the sensor was to incorporate the ionophore into a mixed self-assembled monolayer of 10-mercaptodecanesulfonate and 11-hydroxy-1-undecanethiol formed on the surface. The voltammetric responses for the thus prepared sensor to the primary cation $\mathrm{Ca}^{2+}$ were observed by using $\left[\mathrm{Fe}(\mathrm{CN})_{6}\right]^{3-14}$ as an electroactive marker. The ionophore was stably immobilized on the electrode surface with the hydrophobic interaction between its alkyl chains and those of the alkanethiol. The introduction of a proper charge density to the electrode surface improved the sensor sensitivity with retaining the selective response to $\mathrm{Ca}^{2+}$ against $\mathrm{Mg}^{2+}$ with concentrations above $10^{-4} \mathrm{M}$.
\end{abstract}

(Received August 28, 2006; Accepted October 24, 2006; Published December 10, 2006)

\section{Introduction}

Since the introduction of ion-channel sensors (ICSs) in 1987, research efforts have been directed toward the use of this voltammetric technique for the detection of various ions and molecules. ${ }^{2-5}$ In the method, receptors possessing thiol moieties have been used and immobilized on gold electrode surfaces via strong S-Au covalent bonds. The mechanism of ICSs is based on charged-analyte-gated permeability changes for electroactive ions (often called markers) across self-assembled monolayers (SAMs) of receptors formed on the electrode surfaces. Binding of analytes to the receptors facilitates or suppresses marker access to the surface and subsequent electron transfer between the electrodes and the markers, as a result of electrostatic attraction or repulsion between the analyte-receptor complexes and the markers, respectively.

ICSs have advantages in detecting highly hydrophilic and multiply charged ions, for example, phosphate, ${ }^{6,7}$ protamine, ${ }^{8}$ heparin, ${ }^{9}$ oligonucleotides, ${ }^{10-14}$ and other biomolecules. ${ }^{15-17} \mathrm{We}$ found that ICSs do not require complete dehydration of the analyte ions from the aqueous phase as reported in previous research, ${ }^{6}$ which allows the detection of hydrophilic ions more easily. We also found that a larger change in the surface charge upon binding of analytes to receptors generally results in a higher sensitivity in the ICSs. For example, for the detection of oligonucleotides, we used gold electrodes modified with mixed SAMs of peptide nucleic acid (PNA) probes and protonated 8amino-1-octanethiol based on a positively charged marker

$\dagger$ To whom correspondence should be addressed.

E-mail: aoki-h@aist.go.jp

Present address: Research Institute for Environmental Management Technology, National Institute of Advanced Industrial Science and Technology (AIST), 16-1 Onogawa, Tsukuba, Ibaraki 305-8569, Japan.
$\left[\mathrm{Ru}\left(\mathrm{NH}_{3}\right)_{6}\right]^{3+} .{ }^{11,12}$ PNA is an analogue of DNA, but its backbone is electrically neutral, unlike that of DNA. Without analyte oligonucleotides bound to the probes, the redox reaction of the marker was hindered due to the positive charge at the electrode surface. Binding of the multiply charged complementary oligonucleotide to the PNA probe monolayer neutralizes the positive charge on the surface, and provides an excess negative charge, thereby facilitating the access of the marker to the electrode surface and its redox reaction.

In this paper, we demonstrate a trial to apply a commercially available ion-selective electrode (ISE) ionophore ETH 1001 to an ICS. ISE ionophores were originally designed for retention in liquid organic membranes, rather than for immobilization on electrode surfaces. ${ }^{18-21}$ To prepare the sensor, a gold electrode was modified with a SAM of alkanethiols and dipped in ionophore solutions to form a layer of ETH 1001. The hydrophobic interaction between alkyl chains of the thiols and those of ETH 1001 (see Fig. 1A) was used to immobilize the ionophore on the electrode surface. A negatively charged thiol 10-mercaptodecanesulfonate was used as one of the components of the SAM and a negatively charged complex $\left[\mathrm{Fe}(\mathrm{CN})_{6}\right]^{3-14}$ was used as a marker. Upon binding of $\mathrm{Ca}^{2+}$ to the ETH 1001 layer, the surface charge of the electrode changed from negative to positive, and hitherto repulsed $\left[\mathrm{Fe}(\mathrm{CN})_{6}\right]^{3-14-}$ became attracted to the surface, resulting in an increase in the observed redox currents. In Fig. 1B, the working principle of the sensors is described. The sensor was capable of measuring the primary cation $\mathrm{Ca}^{2+}$ selectively against $\mathrm{Mg}^{2+}$ with concentrations above $10^{-4} \mathrm{M}$.

\section{Experimental}

\section{Reagents and chemicals}

ETH 1001 was purchased from Fluka Chemie AG (Buchs, Switzerland). 11-Hydroxy-1-undecanethiol was purchased from 
A

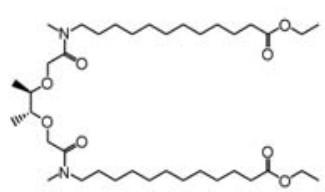

B

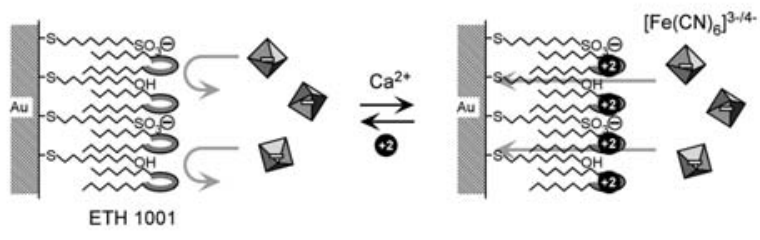

Fig. 1 (A) Chemical structure of ETH 1001. (B) Working principle of the ion-channel sensor for $\mathrm{Ca}^{2+}$ based on ETH 1001 immobilized in a mixed self-assembled monolayer of 10mercaptodecanesulfonate and 11-hydroxy-1-undecanethiol on a gold electrode. Without $\mathrm{Ca}^{2+}$, the negatively charged marker $\left[\mathrm{Fe}(\mathrm{CN})_{6}\right]^{3-14}$ is prevented from accessing the electrode surface due to chargecharge repulsion between the marker and the negatively charged electrode surface. When $\mathrm{Ca}^{2+}$ binds to the ionophore, the negative charge at the surface is decreased, and at higher $\mathrm{Ca}^{2+}$ concentrations, the electrode surface becomes even positively charged. The marker can easily access the surface and its redox reaction is thereby facilitated.

Dojindo Laboratory (Kumamoto, Japan). 10-Mercaptodecanesulfonate sodium salt was synthesized according to previous descriptions. ${ }^{22,23}$ All reagents were of the highest grade commercially available and were used without further purification. Deionized and charcoal-treated water (specific resistance $>18.2 \mathrm{M} \Omega \mathrm{cm}$ ), obtained with a Milli-Q reagentgrade water system (Millipore, Bedford, MA, USA), was used for preparing all of the aqueous solutions. Electrochemical experiments were performed using a CV-50W electrochemical analyzer (Bioanalytical Systems (BAS), West Lafayette, IN, USA) carried out at room temperature with a three-electrode configuration consisting of a $\mathrm{Ag} / \mathrm{AgCl}$ reference electrode, a platinum auxiliary electrode, and the prepared sensor as a working electrode. All potentials were measured versus the $\mathrm{Ag} / \mathrm{AgCl}$ reference electrode.

\section{Preparation of electrodes}

Gold disk electrodes ( $2 \mathrm{~mm}^{2}$ area, BAS) were used for all of the experiments. The electrode was polished with wet 0.3 and $0.05 \mu \mathrm{m}$ alumina suspensions (Micropolish ${ }^{\circledR}$ II deagglomerated alumina; Buehler, Lake Bluff, IL) on a felt pad for at least 10 min, rinsed repeatedly with water, and finally cleaned in a sonicator. The polished electrode was then dipped in a $0.5 \mathrm{M}$ $\mathrm{KOH}$ aqueous solution deoxygenated by purging with argon gas for at least $15 \mathrm{~min}$; the potential was cycled between -400 and $-1200 \mathrm{mV}$ until cyclic voltammograms showed no further changes, indicating that the electrode surface was perfectly clean. To check the purity of the electrode surface, the electrode was scanned over the potential range of 0 to +1600 $\mathrm{mV}$ in a $1 \mathrm{M} \mathrm{H}_{2} \mathrm{SO}_{4}$ aqueous solution until a constant voltammogram (typical of clean polycrystalline gold) ${ }^{24}$ was obtained. The electrochemically polished electrode was soaked in a DMSO solution containing 10-mercaptodecanesulfonate and 11-hydroxy-1-undecanethiol (1:0, 1:1, 1:3, or 1:9) at room temperature for $2.5 \mathrm{~h}$ for surface modification. The total concentration of these two thiols was kept constant at $10 \mathrm{mM}$. The short-time self-assembly of $2.5 \mathrm{~h}$ was expected to decrease the alkanethiol concentration on the electrodes and to reduce the steric hindrance of the thiol SAMs to the marker reaction on the electrode surfaces. After washing with DMSO and ethanol, the electrode was soaked overnight in a $10 \mathrm{mM}$ ethanolic solution of ETH 1001, then washed with ethanol and water. All of the electrodes were stored in water at $4^{\circ} \mathrm{C}$ until use.

\section{Electrochemical measurements}

Cyclic voltammetry (scan rate of $100 \mathrm{mV} \mathrm{s}^{-1}$ ) and square wave voltammetry (step potential of $5 \mathrm{mV}$, square wave frequency of $20 \mathrm{~Hz}$, and square wave amplitude of $50 \mathrm{mV}$ ) were performed as electrochemical measurements. Electrolyte solutions of $0.1 \mathrm{M}$ Tris- $\mathrm{HNO}_{3}$ (adjusted to $\mathrm{pH} 7.4$ by $\mathrm{HNO}_{3}$ ) containing $0.50 \mathrm{mM} \mathrm{K}_{3}\left[\mathrm{Fe}(\mathrm{CN})_{6}\right]$ and $0.50 \mathrm{mM} \mathrm{K}_{4}\left[\mathrm{Fe}(\mathrm{CN})_{6}\right]$ or containing $1.0 \mathrm{mM}\left[\mathrm{Ru}\left(\mathrm{NH}_{3}\right)_{6}\right] \mathrm{Cl}_{3}$ as the electroactive marker were used. The solutions were deoxygenated by purging with argon gas for at least $15 \mathrm{~min}$ prior to the measurements. The analyte concentrations in the solutions were changed by adding aliquots of concentrated analyte solutions. For a study of the sensor responses, the obtained voltammograms were evaluated in terms of the currents at the peak potential $\left(E_{\mathrm{p}}{ }^{\circ}\right)$ in the voltammograms measured in $1.0 \times 10^{-2} \mathrm{M}$ solutions of the analytes.

\section{Results and Discussion}

Figure 2A-1 shows square wave voltammograms of the $\left[\mathrm{Fe}(\mathrm{CN})_{6}\right]^{3-4-}$ marker for an electrode modified with ETH 1001 and a SAM of 10-mercaptodecanesulfonate, where irreversible and almost flat voltammograms were observed. The dependence of the currents at $E_{\mathrm{p}}{ }^{\circ}$ in the voltammograms on the $\mathrm{Ca}^{2+}$ concentration is shown in Fig. 2A-2, where the value of $E_{\mathrm{p}}{ }^{\circ}$ is defined as the peak potential in the voltammogram measured in a buffer solution containing $1.0 \times 10^{-2} \mathrm{M}$ of $\mathrm{Ca}^{2+}$. These results indicate that the redox reaction of the marker on the electrode surface is almost completely suppressed within this concentration range. The positive shift of the value of $E_{\mathrm{p}}{ }^{\circ}$ from $0.225 \mathrm{~V}$ (for a bare gold electrode, data not shown) to $0.325 \mathrm{~V}$ also indicates the suppression of the marker reaction on the electrode surface. In general, the presence of long alkyl chains on the electrode surface and the electrostatic repulsion between the marker and the electrode surface account for the hindrance of the marker reaction. In Fig. 2A-2, inset, cyclic voltammograms of $\left[\mathrm{Fe}(\mathrm{CN})_{6}\right]^{3-14-}$ and $\left[\mathrm{Ru}\left(\mathrm{NH}_{3}\right)_{6}\right]^{3+}$ for this electrode are shown, where the former is quasi-reversible (the peak potential separation: $89 \mathrm{mV}$ ) and the latter is completely irreversible. This selective inhibition of the redox reaction of $\left[\mathrm{Fe}(\mathrm{CN})_{6}\right]^{3-14}$ is attributed to the electrostatic repulsion caused by the negatively charged electrode surface, rather than the steric hindrance caused by the long alkyl chains on the surface. This suggests that the density of 10-mercaptodecanesulfonate in the SAM was excessively high, so that the surface negative charge was not completely cancelled by the binding of $\mathrm{Ca}^{2+}$ to ETH 1001 .

In order to decrease this surface charge, we used mixed SAMs of 10-mercaptodecanesulfonate and 11-hydroxy-1undecanethiol. 11-Hydroxy-1-undecanethiol was expected to function to dilute the surface concentration of 10mercaptodecanesulfonate. Because the chain length of 11hydroxy-1-undecanethiol is nearly equal to that of 10mercaptodecanesulfonate ( $19 \AA$ and $19.5 \AA$ for the former thiol and the latter one, respectively, estimated from CPK models $)^{25}$ and ETH 1001 is electrically neutral, it was thought that the use of 11-hydroxy-1-undecanethiol would have no influence on incorporation of ETH 1001 into the mixed SAMs. The dependence of square wave voltammograms on the $\mathrm{Ca}^{2+}$ 
A 1

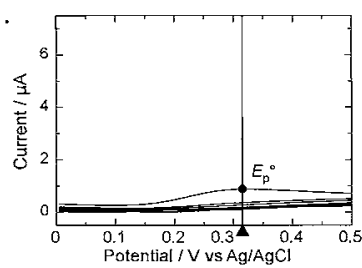

B 1.

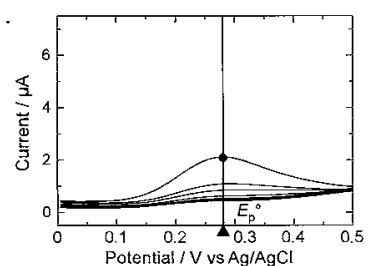

C 1 .

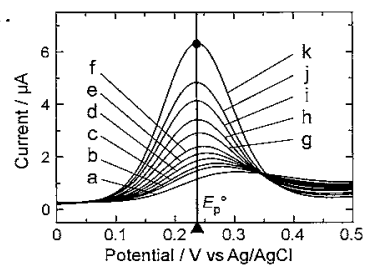

D 1.

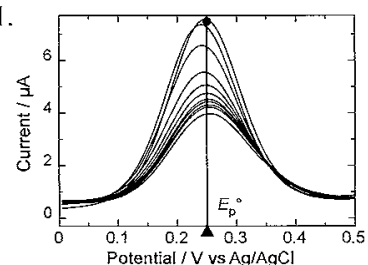

2 .

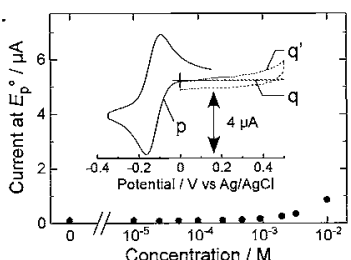

2.
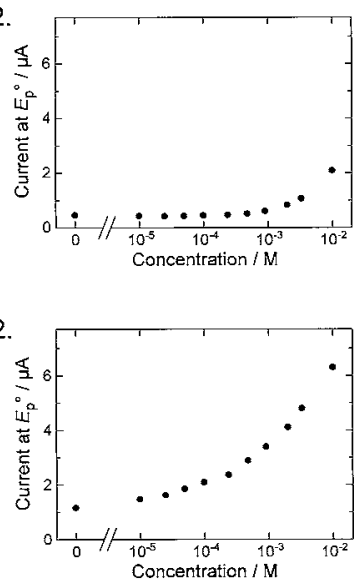

2.

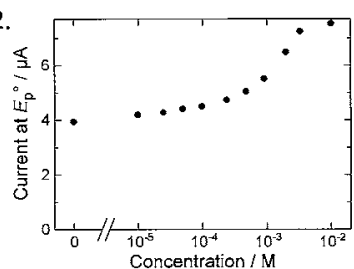

Fig. 2 (1) Typical square wave voltammograms of $\left[\mathrm{Fe}(\mathrm{CN})_{6}\right]^{3-14}$ and (2) dependence of currents at potentials of $E_{\mathrm{p}}{ }^{\circ}$ in the voltammograms on concentrations of $\mathrm{Ca}^{2+}$ ((a) 0 , (b) $1.0 \times 10^{-5}$, (c) $2.5 \times 10^{-5}$, (d) $5.0 \times 10^{-5}$, (e) $9.9 \times 10^{-5}$, (f) $2.4 \times 10^{-4}$, (g) $4.8 \times 10^{-4}$, (h) $9.1 \times 10^{-4}$, (i) $2.0 \times 10^{-3}$, (j) $3.3 \times 10^{-3}$, and (k) $1.0 \times 10^{-2} \mathrm{M}$ ) in $0.1 \mathrm{M}$ Tris- $\mathrm{HNO}_{3}$ buffer solutions $(\mathrm{pH}$ 7.4) for gold electrodes modified with ETH 1001-containing mixed monolayers of 10mercaptodecanesulfonate and 11-hydroxy-1-undecanethiol. Their molar ratios in solutions for self-assembly were 1:0 (A), 1:1 (B), 1:3 (C), and 1:9 (D). The total concentrations of these two thiols were kept constant at $10 \mathrm{mM}$. Inset: the cyclic voltammograms measured in a buffer solution containing $\left[\mathrm{Ru}\left(\mathrm{NH}_{3}\right)_{6}\right]^{3+}$ (solid line (p)) and $\left[\mathrm{Fe}(\mathrm{CN})_{6}\right]^{3-14}$ (dashed line $\left(\mathrm{q}\right.$ and $\left.\mathrm{q}^{\prime}\right)$ ). The voltammogram $\mathrm{q}^{\prime}$ illustrates the current in the voltammogram q magnified by 20 times.

concentration for electrodes modified with mixed monolayers of 10-mercaptodecanesulfonate and 11-hydroxy-1-undecanethiol are shown in Figs. 2B-1 and 2C-1, where the molar ratio of these two thiols in solutions for the self-assembly were 1:1 and $1: 3$, respectively. In these two figures, with increasing the $\mathrm{Ca}^{2+}$ concentration, the values of the peak potential in the voltammograms shifted negatively and the voltammograms became more reversible. This is because binding of $\mathrm{Ca}^{2+}$ to ETH 1001 monolayer decreased the negative charge on the electrode surface and allowed the marker to access the surface more easily. Decreasing the surface density of 10mercaptodecanesulfonate also produced more reversible voltammograms. The observed increase in the reversibility of voltammograms was attributed to the decrease in the surface negative charge caused by $\mathrm{Ca}^{2+}$ binding to the surface-confined ETH 1001 and by decreasing the surface density of the negatively charged thiol. In Fig. 2B-2, the change in current is

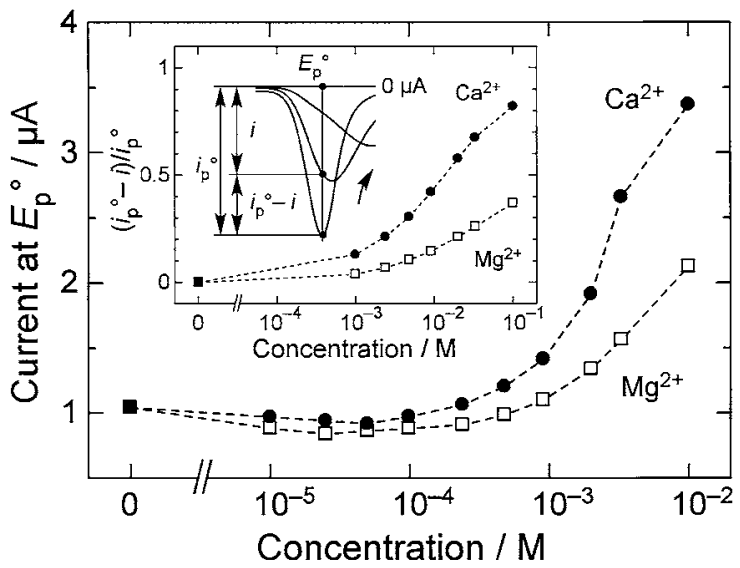

Fig. 3 Dependence of currents at a potential of $E_{\mathrm{p}}{ }^{\circ}$ in square wave voltammograms of $\left[\mathrm{Fe}(\mathrm{CN})_{6}\right]^{3-4-}$ or $\left[\mathrm{Ru}\left(\mathrm{NH}_{3}\right)_{6}\right]^{3+}$ for gold electrodes modified with ETH 1001-containing mixed monolayers (the same electrodes as those used in Fig. 2C) (main) or 11-hydroxyundecanethiol monolayers (inset) measured in buffer solutions on the concentrations of $\mathrm{Ca}^{2+}$ (filled circle) and $\mathrm{Mg}^{2+}$ (open square), respectively. In the inset, the ratios between the peak current at $E_{\mathrm{p}}{ }^{\circ}$ $\left(i_{\mathrm{p}}{ }^{\circ}\right)$ and the current decrease $\left(i_{\mathrm{p}}{ }^{\circ}-i\right)$ are plotted to normalize the signal intensity. Other experimental conditions are the same as those in Fig. 2. The plotted values are the average of the observed values. The relative standard deviation at $5.0 \times 10^{-5} \mathrm{M}$ of $\mathrm{Mg}^{2+}$ was $16.8 \%(n$ $=3$ ) as a typical value.

observed from $\mathrm{ca} \cdot 10^{-3} \mathrm{M}$ of $\mathrm{Ca}^{2+}$, whereas in Fig. $2 \mathrm{C}-2$, the change is observed from $\mathrm{ca} \cdot 10^{-4} \mathrm{M}$ of $\mathrm{Ca}^{2+}$. The responses were observed at lower $\mathrm{Ca}^{2+}$ concentrations. ETH 1001 is stably immobilized on the gold surfaces by hydrophobic intermolecular van der Waals forces between its alkyl chains and those of the alkanethiols composing the SAMs. The sensors prepared in this study were stable without any major observable changes in the performance as long as they were stored in a $0.1 \mathrm{M}$ Tris- $\mathrm{HNO}_{3}$ buffer solution; they could be used for measurements of over 390 voltammograms.

Moreover, a case with a lower molar ratio of 10mercaptodecanesulfonate to 11-hydroxy-1-undecanethiol was investigated, resulting in reduction of the current dependence on the $\mathrm{Ca}^{2+}$ concentration (Fig. 2D). The voltammograms became more reversible, because the effect of the surface negative charge became smaller due to the decrease in the surface concentration of 10-mercaptodecanesulfonate. The introduction of a proper charge density to the electrode surface is necessary to enhance the dependence of the observed current on the $\mathrm{Ca}^{2+}$ concentration.

As described above, the sensor prepared from the solution containing the charged thiol and the electrically neutral thiol with a molar ratio of 1:3 showed the best sensitivity to $\mathrm{Ca}^{2+}$ among the sensors examined. To investigate the selectivity of this sensor, the sensor responses to $\mathrm{Ca}^{2+}$ and $\mathrm{Mg}^{2+}$ were compared (Fig. 3, main). The plotted values are the average of the observed values. The relative standard deviation at $5.0 \times$ $10^{-5} \mathrm{M}$ of $\mathrm{Mg}^{2+}$ was $16.8 \%(n=3)$, a typical value in this experiment. The selective response to $\mathrm{Ca}^{2+}$ was observed from ca. $10^{-4} \mathrm{M}$, while a smaller response to $\mathrm{Mg}^{2+}$ was also noted from $c a \cdot 10^{-3}$ M. $\mathrm{Na}^{+}$and $\mathrm{K}^{+}$were not detected within this concentration range. In a previous study on oligonucleotide sensors, it was demonstrated that oligonucleotides bound nonselectively to the protonated amine group of the SAM at the electrode surface regardless of their sequences. ${ }^{11}$ In the present system studied here, it is possible that $\mathrm{Ca}^{2+}$ and $\mathrm{Mg}^{2+}$ bind non- 
selectively to the sensor surface due to the sulfonate moiety of the negatively charged thiol. In order to examine the possibility of the effect of the sulfonate moiety on the sensor responses, therefore, an electrode modified with ETH 1001 and a SAM of 11-hydroxy-1-undecanethiol, without 10-mercaptodecanesulfonate, was also studied. $\left[\mathrm{Ru}\left(\mathrm{NH}_{3}\right)_{6}\right]^{3+}$ was used as a marker and the sensor response was evaluated by square wave voltammetry (Fig. 3, inset), where the ratios between the peak current at $E_{\mathrm{p}}{ }^{\circ}\left(i_{\mathrm{p}}{ }^{\circ}\right)$ and the current decrease $\left(i_{\mathrm{p}}{ }^{\circ}-i\right)$ were plotted to normalize the signal intensity. The response to $\mathrm{Ca}^{2+}$ was shown at concentrations higher than $10^{-3} \mathrm{M}$, while that to $\mathrm{Mg}^{2+}$ at concentrations above $10^{-2} \mathrm{M}$. The figure shows that, without the negatively charged thiol, the selectivity of $\mathrm{Ca}^{2+}$ against $\mathrm{Mg}^{2+}$ was apparently increased, although the effect was small. This shows that the sulfonate moiety of the charged thiol might be responsible for the non-selective adhesion of the ions to the SAM. That is, while the use of the charged thiol is effective for the sensor sensitivity, the thiol also affects the sensor selectivity: A trade-off exists between the sensitivity and the selectivity. Therefore, to balance the selectivity with the sensitivity, optimization of the surface density of the charged thiol is important. In terms of not only improving the sensitivity but also retaining the selectivity to $\mathrm{Ca}^{2+}$, the sensor prepared in this study is thought to be one of the optimum results.

\section{Conclusions}

We observed that ETH 1001 was stably immobilized on gold electrode surfaces modified with SAMs of 10mercaptodecanesulfonate and 11-hydroxy-1-undecanethiol via hydrophobic intermolecular van der Waals forces between the alkyl chains of ETH 1001 and those of the alkanethiols. By optimizing the density of the charged thiol on the electrode surfaces based on the mixed SAMs, the sensor response to $\mathrm{Ca}^{2+}$ was enhanced and a higher sensitivity was yielded. Although introduction of the charged thiol to the electrode surface improved the sensor response to $\mathrm{Mg}^{2+}$ as well as that to $\mathrm{Ca}^{2+}$, the selective detection of $\mathrm{Ca}^{2+}$ against $\mathrm{Mg}^{2+}$ was demonstrated.

As expected in Experimental, the short-time self-assembly for forming alkanethiol SAMs contributed to the reduction of the steric hindrance to the marker reaction, where stable immobilization of the ionophore molecules on the electrode surfaces was also realized.

\section{Acknowledgements}

This work has been supported by a fellowship from the Japan Society for the Promotion of Science (JSPS).

\section{References}

1. M. Sugawara, K. Kojima, H. Sazawa, and Y. Umezawa, Anal. Chem., 1987, 59, 2842.

2. P. Bühlmann, H. Aoki, K. P. Xiao, S. Amemiya, K. Tohda, and Y. Umezawa, Electroanalysis, 1998, 10, 1149.

3. L. Luo, X. Yang, and E. Wang, Anal. Lett., 1999, 32, 1271.

4. M. Sugawara, A. Hirano, P. Bühlmann, and Y. Umezawa, Bull. Chem. Soc. Jpn., 2002, 75, 187.

5. Y. Umezawa and H. Aoki, Anal. Chem., 2004, 76, 320A.

6. K. P. Xiao, P. Bühlmann, and Y. Umezawa, Anal. Chem., 1999, 71, 1183.

7. H. Aoki, K. Hasegawa, K. Tohda, and Y. Umezawa, Biosens. Bioelecton., 2003, 18, 261.

8. V. P. Y. Gadzekpo, K. P. Xiao, H. Aoki, P. Bühlmann, and Y. Umezawa, Anal. Chem., 1999, 71, 5109.

9. V. P. Y. Gadzekpo, P. Bühlmann, K. P. Xiao, H. Aoki, and Y. Umezawa, Anal. Chim. Acta, 2000, 411, 163.

10. H. Aoki, P. Bühlmann, and Y. Umezawa, Electroanalysis, 2000, 12, 1272.

11. H. Aoki and Y. Umezawa, Electroanalysis, 2002, 14, 1405.

12. H. Aoki and Y. Umezawa, Analyst, 2003, 128, 681.

13. H. Aoki and H. Tao, Analyst, 2005, 130, 1478.

14. A. Han, T. Takarada, T. Shibata, M. Nakayama, and M. Maeda, Anal. Sci., 2006, 22, 663.

15. H. Kuramitz, K. Sugawara, and S. Tanaka, Electroanalysis, 2000, 12, 1299.

16. Y. Katayama, Y. Ohuchi, H. Higashi, Y. Kudo, and M. Maeda, Anal. Chem., 2000, 72, 4671.

17. M. Murata, K. Yano, S. Kuroki, T. Suzutani, and Y. Katayama, Anal. Sci., 2003, 19, 1569.

18. Y. Umezawa, in "CRC Handbook of Ion-selective Electrodes: Selectivity Coefficients", 1990, CRC Press, Boca Raton, FL.

19. Y. Umezawa, P. Bühlmann, K. Umezawa, K. Tohda, and S. Amemiya, Pure Appl. Chem., 2000, 72, 1851.

20. Y. Umezawa, K. Umezawa, P. Bühlmann, N. Hamada, H. Aoki, J. Nakanishi, M. Sato, K. P. Xiao, and Y. Nishimura, Pure Appl. Chem., 2002, 74, 923.

21. Y. Umezawa, P. Bühlmann, K. Umezawa, and N. Hamada, Pure Appl. Chem., 2002, 74, 995.

22. I. Turyan and D. Mandler, J. Am. Chem. Soc., 1998, 120, 10733.

23. J.-C. Lin and W.-H. Chuang, J. Biomed. Mater. Res., 2000, $51,413$.

24. R. Woods, in "Electroanalytical Chemistry", ed. A. J. Bard, 1976, Vol. 9, Marcel Dekker, NY.

25. W. L. Koltun, Biopolymers, 1965, 3, 665. 